\title{
Combinatorial Investigation of Ferromagnetic Shape-Memory Alloys in the Ni-Mn-Al Ternary System Using a Composition Spread Technique
}

\author{
Olugbenga O. Famodu ${ }^{1}$, Jason Hattrick-Simpers ${ }^{1}$, Maria Aronova ${ }^{2}$, Kao-Shuo Chang ${ }^{1}$, \\ Makoto Murakami ${ }^{1}$, Manfred Wuttig ${ }^{1}$, Teiko Okazaki ${ }^{3}$, Yasubumi Furuya ${ }^{3}$, Lee A. Knauss ${ }^{4}$, \\ Leonid A. Bendersky ${ }^{5}$, Frank. S. Biancaniello ${ }^{5}$ and Ichiro Takeuchi ${ }^{1,2 *}$
}

${ }^{1}$ Small Smart Systems Center, Department of Materials Science and Engineering, University of Maryland, College Park, MD 20742, USA

${ }^{2}$ Center for Superconductivity Research, Department of Physics, University of Maryland, College Park, MD 20742, USA

${ }^{3}$ Faculty of Science and Technology, Hirosaki University, Hirosaki, Japan

${ }^{4}$ Neocera, Inc., 10000 Virginia Manor Road, Beltsville, MD 20705, USA

${ }^{5}$ Materials Science and Engineering Laboratory, National Institute of Standards and Technology (NIST),

100 Bureau Drive, Gaithersburg, MD 20899-8555, USA

Using a thin-film composition spread technique, we have mapped the phase diagram of the Ni-Mn-Al ternary system in search of ferromagnetic shape-memory alloys (FMSA). A characterization technique that allows detection of martensitic transitions by visual inspection using micromachined cantilever arrays was combined with quantitative magnetization mapping using scanning superconducting quantum interference device (SQUID) microscopy. A large compositional region in the Al deficient part of the phase diagram was found to be ferromagnetic and reversibly martensitic at room temperature. In addition, in the Al rich region, a new compositional range that displays marked ferromagnetism was found.

(Received October 6, 2003; Accepted November 18, 2003)

Keywords: combinatorial, ferromagnetic shape-memory alloys, composition spreads, thin-film, phase diagram, martensitic transitions, quantitative magnetization mapping, scanning superconducting quantum interference device (SQUID) microscopy, magnetization, NiMnAl

\section{Introduction}

Ferromagnetic shape-memory alloys (FMSAs) are an important class of materials that can be used for applications in magnetomechanical devices and sensors. ${ }^{1)}$ Research in this field has been largely focused on $\mathrm{Fe}-\mathrm{Pd}$ and $\mathrm{Ni}_{2} \mathrm{MnGa}$ due to the large strains induced in these systems by an external magnetic field. ${ }^{2-4)}$ Other alloy systems such as $\mathrm{Co}_{2} \mathrm{NiGa}$ and $\mathrm{Co}_{2} \mathrm{NiAl}$ have also been found to be FMSAs. ${ }^{5,6)}$ Previously, we have demonstrated functional mapping of the Ni-Mn-Ga ternary system and found a large previously unexplored region of the ternary phase diagram that is ferromagnetic and reversibly martensitic. ${ }^{7)}$ Partly due to the brittleness of these known materials, there is a continuing interest in finding other FMSAs. ${ }^{8,9)}$ The Ni-Mn-Al alloy system is considered to be a good candidate because it does not contain $\mathrm{Ga}$ and the alloys are less brittle than Ni-Mn-Ga. ${ }^{6,9-11,31,32)}$ In this work, we have mapped the physical properties of the Ni-Mn-Al ternary system with respect to ferromagnetism and martensitic transitions using the composition spread technique.

\section{Experimental Techniques}

We have developed thin-film based high-throughput fabrication and detection techniques for exploring FSMAs. ${ }^{7,27)}$ The screening technique employs micromachined arrays of mechanical cantilever libraries to detect structural transformation in thin-film composition spreads, combined with room-temperature quantitative remnant magnetization mapping using a scanning SQUID microscope. In

*Corresponding author, E-mail: takeuchi@squid.umd.edu addition, a scanning $\mathrm{x}$-ray microdiffractometer is used to map the structure. ${ }^{7)}$

Natural composition spreads of the Ni-Mn-Al system were deposited using an ultra high-vacuum magnetron co-sputtering system (with a base pressure in the range of $10^{-7} \mathrm{~Pa}$ ) on $76.2 \mathrm{~mm}$ (three-inch) diameter (100) Si wafers. Three $38.1 \mathrm{~mm}$ (1.5-inch) diameter guns are placed parallel and adjacent to each other in a triangular configuration. Figure 1 illustrates our composition spread deposition scheme. Each gun is housed in a $50.8 \mathrm{~mm}$ (2-inch) long chimney which (a)

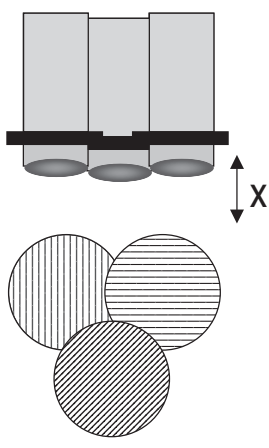

(b)

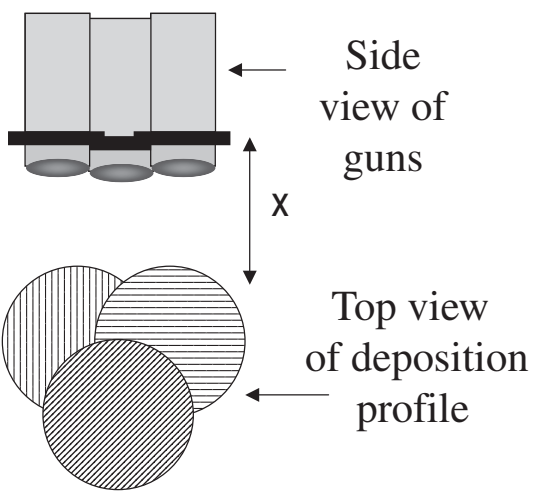

Fig. 1 Schematic of co-sputtering of natural ternary composition spread using parallel gun geometry. Three $38.1 \mathrm{~mm}$ (1.5-inch)-diameter guns are placed parallel and adjacent to each other in a triangular configuration. By adjusting the distance $(\mathrm{x})$, between the substrate and parallel guns we can control the compositional region of the phase diagram covered on the substrate. (a) shows a schematic of deposition pattern for a short distance between the substrate and guns. (b) shows the schematic of deposition pattern at a further distance. 
helps to minimize the cross contamination of the guns. The three targets used in the present experiment were $\mathrm{Ni}, \mathrm{Mn}$ and $\mathrm{Al}$, and both DC and RF sputtering were used. The typical gun power was 50 150 watts, and spread films with thicknesses in the range of $250 \mathrm{~nm}$ to $0.5 \mu \mathrm{m}$ were deposited in $1 \sim 2 \mathrm{~h}$. By adjusting the power applied to each gun and the distance between the guns and the substrate (typically $12 \mathrm{~cm}$ ), different regions of the ternary phase diagram can be mapped. The composition spread wafers were deposited at room temperature followed by an in-situ annealing in vacuum at temperatures in the range of 853-970 K for $2 \mathrm{~h}$. $\mathrm{X}$-ray microdiffraction of the fabricated films was performed using the $\omega$-scan mode of a D8 DISCOVER (Bruker-AXS for combinatorial screening). Wavelength dispersive spectroscopy (WDS) was used to accurately map the composition spread of every wafer.

For rapid characterization of magnetic properties, we used a room temperature scanning SQUID microscope, ${ }^{12)}$ which provides mapping of local magnetic field emanating from samples at room temperature. Prior to the scan, the spread wafer is magnetized in an in-plane direction. Figure 2(a) is a magnetic field image of a Ni-Mn-Al spread wafer. This spread was annealed at $973 \mathrm{~K}$ for $2 \mathrm{~h}$. Variation in the strength of the magnetic field, as a function of composition is evident. In this ternary system, we have consistently observed two distinct compositional regions, which showed ferromagnetism at room temperature. We have used a numerical algorithm to directly convert the field distribution information into quantitative remanent magnetization. ${ }^{13)}$ The values of remanent magnetization extracted here are consistent with those obtained by a vibrating sample magnetometer (VSM) on separate individual composition samples measured at room temperature. The result of this calculation was then combined with the composition mapping to obtain a room-temperature magnetic phase diagram (Fig. 2(b)).

In order to map the regions of shape memory alloys and their transition temperatures for the entire spread, we have micromachined arrays of cantilevers and deposited the composition spreads directly on the array wafers (Fig. 3(a)). In order to study the thermally induced actuation of the entire cantilever array simultaneously by visual inspection, we developed a method that works on the simple principle that individual cantilevers with metallic films deposited on them behave as concave mirrors. During a transition, stress-induced actuation on a cantilever results in a sudden change in the radius of the "mirror", and an image reflected off of the cantilevers responds very sensitively as the concavity of the mirrors change. By monitoring the change in the image as a function of temperature, composition regions undergoing a transition can be readily discerned. ${ }^{7)}$

Figure 3(a) is a photograph of a spread deposited on a cantilever array, which is reflecting an image of a series of colored lines. The measurement consists of recording the image projected on cantilever arrays as the temperature is varied. From the cantilevers displaying transitions, another phase diagram is constructed (Fig. 3(b)) which shows the composition regions that undergo martensitic transitions, and the corresponding transition temperatures. Because of the finite size of individual cantilevers, there is compositional

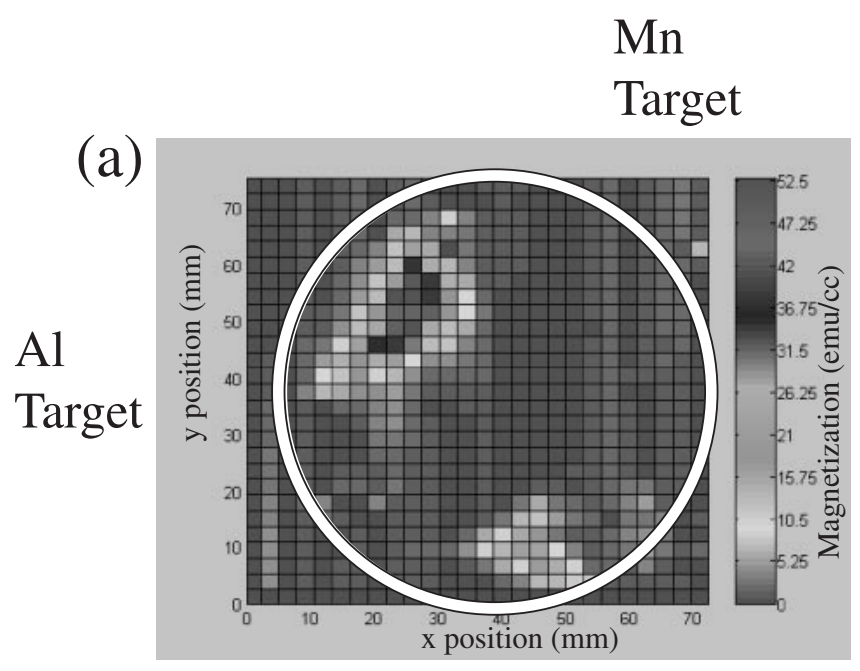

$\mathrm{Ni}$

Target

(b)

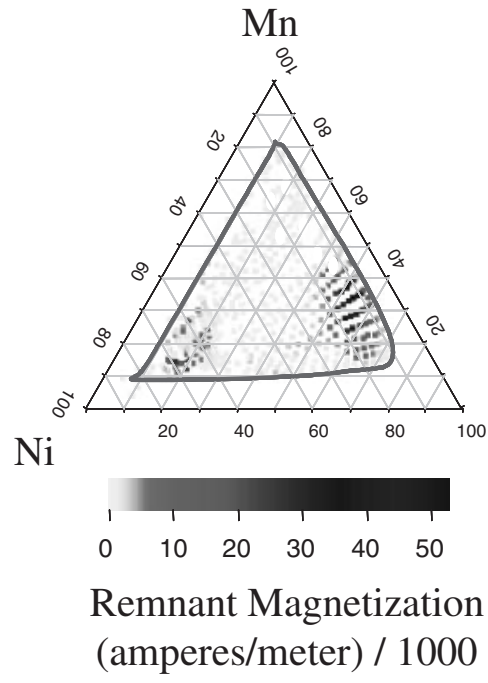

Fig. 2 Scanning SQUID microscope mapping of magnetic properties at room temperature. (a) Scanning SQUID image of a N-Mn-Al spread wafer. The spread is patterned into arrays of $1.75 \mathrm{~mm} \times 1.75 \mathrm{~mm}$ square grids so that the microscope can detect variation of magnetization in the in-plane magnetized samples. The SQUID microscope is sensitive to magnetic poles. The separation between the SQUID and the sample is about $0.3 \mathrm{~mm}$, which defines the spatial resolution of the measurement. The circle is the outline of the wafer. This wafer was annealed at $973 \mathrm{~K}$. (b) Room-temperature magnetic phase diagram of Ni-Mn-Al deduced from (a). The region inside the curve is the compositional region mapped on the spread wafer.

variation on each cantilever. A typical thermal hysteresis width of a martensitic transition observed here is about $50 \mathrm{~K}$, and this is partly attributed to the compositional variation within each cantilever. WDS was performed at three positions along the length of each cantilever. Since it is difficult to distinguish the martensitic transformations of different regions on a single cantilever, for the purpose of mapping, we have labeled three compositions with one transition temperature observed for the cantilever. In Fig. 3 (b), we plot results from one composition spread wafer. This spread was annealed at $853 \mathrm{~K}$ for $2 \mathrm{~h}$. A clear trend emerges, and the general region that undergoes martensitic transitions 
(a)

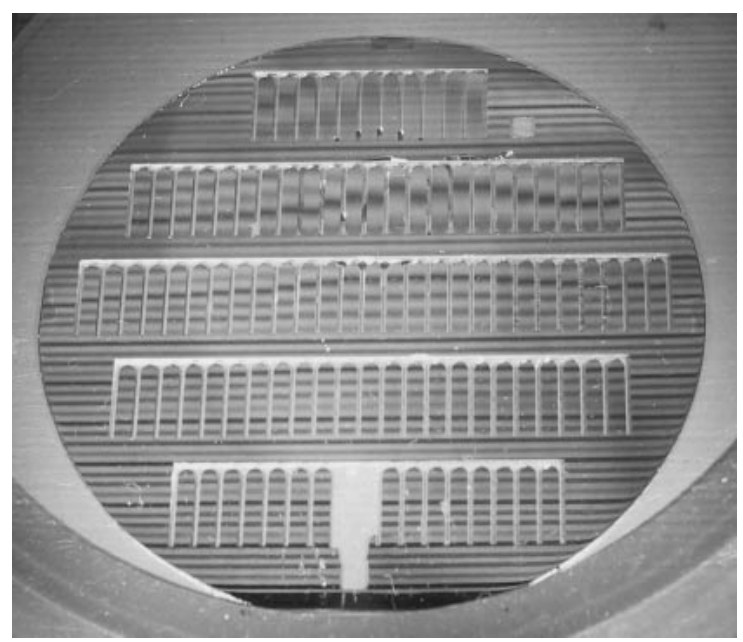

(b)

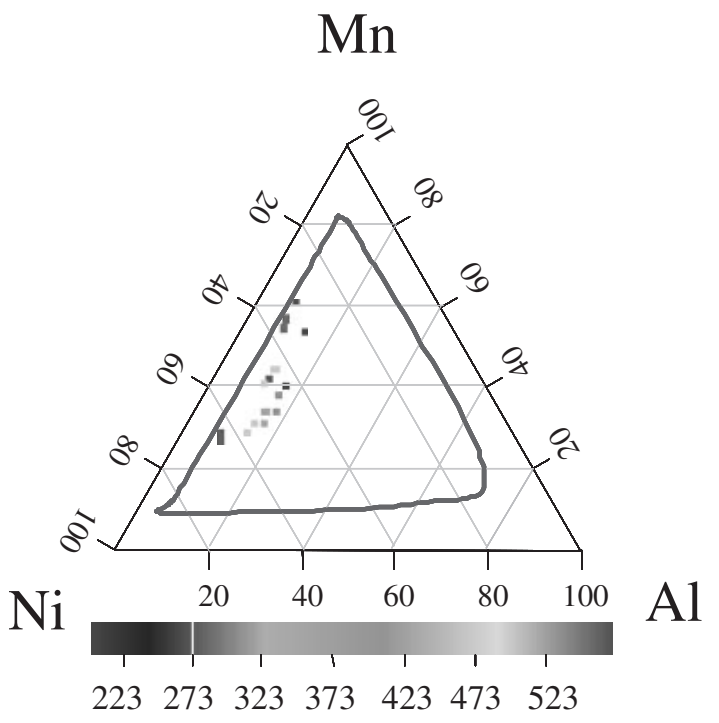

Martensite Start Transition, T/K

Fig. 3 Micromachined cantilever arrays used for detection of shapememory alloys. (a) A photograph of a spread deposited on a cantilever library during the temperature-dependent measurement. A typical cantilever has an area of $2 \mathrm{~mm}$ by $8.5 \mathrm{~mm}$ and thickness of $60 \mu \mathrm{m}$. The lines are a reflection of an image with colored lines held at an incident angle over the wafer. The shift in the line positions of the reflected image as a function of temperature is used to detect small changes in the local curvature of the cantilever. (b) Mapping of the compositional regions on the phase diagram that displayed martensitic transformation on one wafer, which was annealed at $853 \mathrm{~K}$. Martensite start temperature is plotted. The region inside the curve is the compositional region mapped on the spread wafer.

can be easily seen from the phase diagram. The exact martensitic transition temperatures are known to depend on many factors such as atomic ordering, microstructure and the residual stress in the film. ${ }^{14-16)}$ Reported values of the martensitic transition temperatures of Ni-Mn-Al samples in the literature also vary widely, depending on the composition and processing conditions. For this reason, we focus on the trend of the martensitic transition temperature as a function of compositional variation, rather than on the exact transition temperatures.

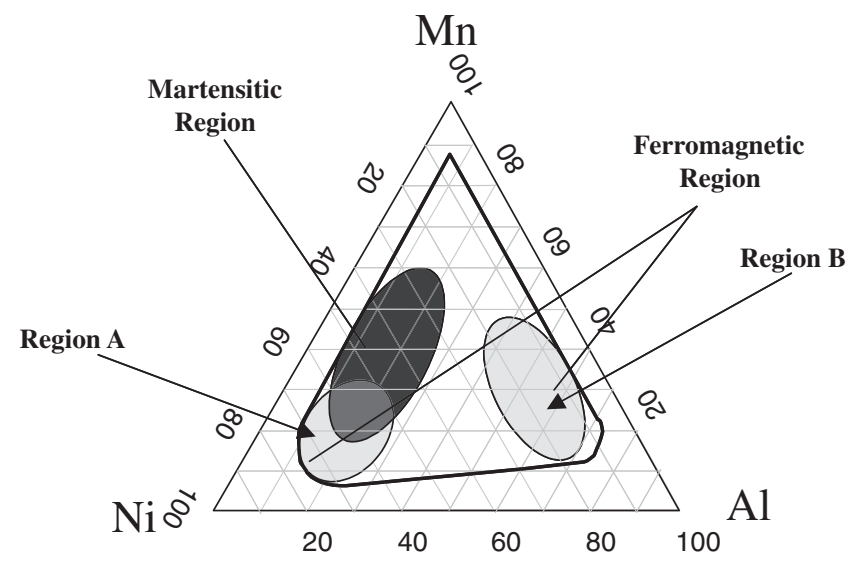

Fig. 4 Trends observed in spread pictured in Fig. 2(b) and the cantilever library in Fig. 3(b) are summarized. Ferromagnetism was observed in two regions. Martensites are observed in a compositional region similar to the region in the Ni-Mn-Ga system.

\section{Discussion}

There have been a number of reports on the investigation of properties of different regions of the Ni-Mn-Al ternary system over the years. Most of the work has been on bulk alloy samples. Recently, there have also been reports on $\mathrm{Ni}$ $\mathrm{Mn}-\mathrm{Al}$ thin films. ${ }^{2,17)}$ There are some discrepancies in the reported magnetic properties of Ni-Mn-Al alloys. This appears to originate from the sensitivity of the properties on thermal treatment, which varies among different studies. ${ }^{18)}$ Different thermal treatments naturally affect the degree of crystallization as well as the degree of disorder in samples.

Figure 4 summarizes our findings in the present experiment. There are two compositional regions that display ferromagnetism in this ternary system as can be seen in Fig. 2(b). In Region $\mathrm{A}$, the composition range encompasses $\mathrm{Ni}$ (50-90 at\%), Mn (10-40 at\%), and Al (8-23 at\%) and in Region B the composition range covers $\mathrm{Ni}$ (9-25 at\%), Mn (15-50 at\%), and $\mathrm{Al}$ (40-70 at\%). In Region A, the magnitude of remnant magnetization becomes stronger as one goes towards higher Ni contents. The maximum remanent magnetization found here in Region $\mathrm{A}$ was in the range of $\sim 40000 \mathrm{~A} / \mathrm{m}$ per meter $(\sim 40 \mathrm{emu} / \mathrm{cc})$ for a nominal composition of $\mathrm{Ni}_{76} \mathrm{Mn}_{17} \mathrm{~A}_{7}$.

It is well-known that order-disorder plays an important role in determining ferromagnetic and antiferromagnetic (AFM) properties of materials as has been reported in previous work. ${ }^{7,19,28,30)}$ It is known that the nominal $\mathrm{Ni}_{2} \mathrm{MnAl}$ composition can display ferromagnetism or antiferromagnetism depending on processing conditions, and in turn on the degree of order/disorder. ${ }^{17,19,33)}$ We believe that this is the main reason for the discrepancy between our findings in Region A and other reports.

In principle, different phases and crystal structures exist across our spreads, thus the atomic order parameter after a specific heat treatment is different for various regions. Previously, we have found that our heat treatment results predominantly in equilibrium phases. Thus, we believe that the phases present in our spreads have a relatively high degree of ordering. We are currently investigating the details of the effect of order/disorder by fabricating spreads with 
different cooling procedures so that different degrees of order/disorder will occur in our films.

We find that the compositional trend of martensites here appears to be very similar to what we found in the Ni-Mn-Ga system. ${ }^{7)}$ This region stretches from the Heusler composition of $\mathrm{Ni}_{2} \mathrm{MnAl}$ to the Al-deficient region (Fig. 4). As one moves towards the more Al-deficient region, the martensite transition temperature increases (Fig. 3(b)). This region appears to correspond exactly with the region where the $\beta / \mathrm{B} 2$ parent phase has been reported. ${ }^{9,10,18,20,29,30)}$ An X-ray microbeam diffraction scan of the entire spread library indicates that most of the regions have diffractions consistent with the L $2{ }_{1}$ or the B2 phase. This is in agreement with other published work. $^{8-10,17,20-24)}$ Previously, Kainuma, et al. ${ }^{9,20)}$ also mapped the martensites in this region and reported a similar trend.

The finding here seems to indicate the presence of FMSAs in the Al-deficient Ni-rich region of the phase diagram where there is an overlap of compositions displaying martensites and remanent magnetization.

In order to confirm our findings from composition spreads, a bulk sample was made with a nominal composition of $\mathrm{Ni}_{52.5} \mathrm{Mn}_{30.9} \mathrm{Al}_{16.5}$, which is in the ferromagnetic and martensitic region. An ingot of the $\mathrm{Ni}_{52.5} \mathrm{Mn}_{30.9} \mathrm{Al}_{16.6}$ alloy was prepared from manganese, nickel and aluminum by using an arc-melting method in an argon atmosphere. A composition of the alloy was determined by electron microprobe. The bulk sample was annealed for $1 \mathrm{~h}$ at $773 \mathrm{~K}$ and then quenched in water. Figure 5 shows the room temperature X-ray diffraction of the bulk sample. After the anneal, the (220) peak has split into two peaks indicating that the alloy is indeed a martensite. This sample was however found to be mostly paramagnetic down to $4 \mathrm{~K}$. We again attribute the difference in magnetic properties to different degrees of disorder in the bulk sample and our thin film spread. Analysis of the XRD result for the bulk sample also showed that the sample was polycrystalline and orthorhombic. An optical microscope study of a bulk sample has revealed plate like features consistent with presence of martensites.

We have found a large, previously unknown, area of the ternary phase diagram (Region B) that displays marked ferromagnetism. This region contains compositions with remnant magnetizations up to $50000 \mathrm{~A} / \mathrm{m}(50 \mathrm{emu} / \mathrm{cc})$. A qualitative remnant maximum of magnetization $53000 \mathrm{~A} / \mathrm{m}$ (53 emu/cc) is reached at $\mathrm{Ni}_{14} \mathrm{Mn}_{32} \mathrm{Al}_{54}$ on the spread. This

$\mathrm{Ni}_{52.5} \mathrm{Mn}_{31} \mathrm{Al}_{16.5}$

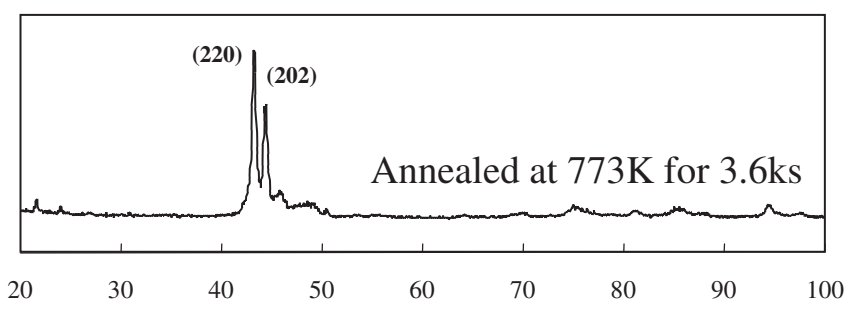

Diffraction Angle, $2 \theta$

Fig. 5 X-Ray diffraction taken at room temperature of a bulk sample after it is annealed at $773 \mathrm{~K}$ for $1 \mathrm{~h}$. was confirmed by measuring a single square sample of the thin film cut from the spread with a VSM.

Near Region B, MnAl is known to have a metastable bct $\tau$ phase, which displays hard ferromagnetic properties. There have been reports of synthesis of this phase in bulk as well as in thin film samples. ${ }^{24-26)}$ In sputtered films, it was found that upon substituting $\mathrm{Ni}$ ions into $\mathrm{MnAl}$, the lattice forms a more stable cubic $\kappa$-phase, which has a $\mathrm{CsCl}$ type structure. ${ }^{26)} \mathrm{It}$ has been reported that the magnetic properties of the $\kappa$-phase varies as the $\mathrm{Ni}$ concentration is increased.

We have discovered a much larger area which displays ferromagnetism that was previously unexplored. The qualitative trend of magnetization we observe for iso-aluminum samples in our spreads, which include the region in which the $\kappa$-phase was reported, is very similar to the reported trend, and there is a rather broad maximum of remnant magnetization being observed for intermediate dopings of $\mathrm{Ni}$. The values of magnetization we observe in this region are of the same order as those in the previous reports. ${ }^{25,26)}$

XRD data of our spread wafers show that in this compositional region the value of the lattice constant is higher than in any other region of the ternary phase diagram. This may explain the occurrence of ferromagnetism: it is consistent with the picture that as the distance between $\mathrm{Mn}$ ions is increased, exchange interaction shifts from anti-ferromagnetic to ferromagnetic. ${ }^{19)}$

\section{Summary}

Using composition spreads, we have found that there are regions where both ferromagnetic and shape-memory properties coexist in the ternary $\mathrm{Ni}-\mathrm{Mn}-\mathrm{Al}$ alloy system. In addition, we observed a large Al-rich region of the phase diagram that shows remanent magnetism. We attribute the discrepancies in magnetic properties between our observations and other reports on different degrees of disorder in the samples. We confirmed that the martensitic composition found in our phase diagram indeed shows XRD consistent with a martensite in a bulk sample. More detailed investigation of individual compositions selected from the regions of interest is currently underway. In addition, further work on XRD phase mapping of the crystal structure is planned.

\section{Acknowledgements}

We acknowledge useful discussions with Cui Jun, S. E. Lofland and F.C. Wellstood. This project was funded by ONR N000140010503, N000140110761, N000140410085 and NSF DMR0231291.

\section{REFERENCES}

1) R. C. O’Handley, S. J. Murray, M. Marioni, H. Nembach and S. M. Allen: J. Appl. Phys. 87 (2000) 4712-4717.

2) R. D. James and M. Wuttig: Philos. Mag. A 77 (1998) 1273-1299.

3) K. Ullakko, J. K. Huang, C. Kantner, R. C. O'Handley and V. V. Kokorin: Appl. Phys. Lett. 69 (1996) 1966-1968.

4) A. Fujita, K. Fukamichi, F. Gejima, R. Kainuma and K. Ishida: Appl. Phys. Lett. 77 (2000) 3054-3056.

5) K. Oikawa, T. Ota, F. Gejima, T. Ohmori, R. Kainuma and K. Ishida: Mater. Trans. 42 (2001) 2472-2475. 
6) C. Craciunescu, Y. Kishi, T. A. Lograsso and M. Wuttig: Scri. Mater. 47 (2002) 258-288.

7) I. Takeuchi, O. O. Famodu, J. C. Read, M. A. Aronova, K.-S. Chang, C. Craciunescu, S. E. Lofland, M. Wuttig, F. C. Wellstood, L. Knauss and A. Orozco: Nature Materials 2 (2003) 180-184.

8) L. Manosa, A. Planes, M. Acet, E. Duman and E. F. Wassermann: J. Appl. Phys. 93 (2003) 8498-8500.

9) R. Kainuma, H. Nakano and K. Ishida: Metall. Mater. Trans. A 27A (1996) 4153-4162.

10) S. Morito, T. Kakeshita, K. Hirata, and K. Otsuka: Acta Mater. 46 (1998) 5377-5384.

11) Y. Sutou, I. Ohnuma, R. Kainuma, and K. Ishida: Metall. Mater. Trans. A 29A (1998) 2225-2227.

12) E. F. Fleet, S. Chatraphorn, F. C. Wellstood, L. A. Knauss and S. M. Green: Rev. Sci. Inst. 72 (2001) 3281-3290.

13) Fleet, E. F. Ph.D. thesis, University of Maryland, 2000.

14) M. Ahlers: Journal de Physique IV, Colloque C8, supplement au Journal de Physique III 5 (1995) C8-71-C8-80.

15) S. Miyazaki and A. Ishida: Mater. Sci. Eng. A273 (1999) 106-133.

16) C. M. Craciunescu, J. Li and M. Wuttig: Scri. Mater. 48 (2003) 65-70

17) X. Y. Dong, J. W. Dong, J. Q. Xie, T. C. Shih, S. McKernan, C. Leighton and C. J. Palmstrom: J. Crys. Growth 254 (2003) 384-389.

18) Ll. Manosa, A. Plans, Ch. Somsen, Ch. Fell and M. Acet: J. Phys. IV France 11 (2001) Pr8-245-249.

19) M. Acet, E. Duman, E. F. Wasserman, L1. Manosa and A. Planes: J. Appl. Phys. 92 (2002) 3867-3871.
20) R. Kainuma, F. Gejima, Y. Sutou, I. Ohnuma and K. Ishida: Mater. Trans., JIM 41 (2000) 943-949.

21) [21] P. L. Potapov, N. A. Polyakova, V. A. Udovenko and E. L. Svistunova: Z. Metallkd. 87 (1996) 33-39.

22) R. C. Taylor and C. C. Tsuei: Solid State Communi. 41 (1982) 503506.

23) Y. Tan, T. Shinoda, Y. Mishima, and T. Suzuki: Mater. Trans. 42 (2001) 464-470.

24) D. P. Hoydick, E. J. Palmiere, and W. A. Soffa: Scr. Mater. 36 (1997) 151-156.

25) J. P. Harbison, T. Sands, R. Ramesh, L. T. Florez, B. J. Wilkens, and V. G. Keramidas: J. Cryst. Growth 111 (1991) 978-983.

26) A. Morisako, N. Koshiro, M. Matsumoto and M. Naoe: J. Appl. Phys. 67 (1990) 5655-5657.

27) J. Soltys: Physica Status Solidi (A) 66 (1981) 485-491.

28) R. M. Bozorth: IEEE Press, New Jersey. Ferromagnetism (1993) 317.

29) T. Shinoda, Y. Mishima and T. Suzuki: Mat. Res. Soc. Symp. Proc. 364 (1995) 573-577.

30) S. Morito and K. Otsuka: Mater. Sci. Engr. A208 (1996) 47-55.

31) T. Kanomata, K. Shirakawa and T. Kaneko: J. Mag. Mag. Mater. 65 (1987) 76-82.

32) W. Deng, R. S. Brussa, G. P. Karwasz and A. Zecca: Mater. Sci. Forum 363-365 (2001) 198-200.

$33)$ K. K. Jee, P. L. Potapov, S. Y. Song and M. C. Shin: Scr. Mater. 36 (1997) 207-212. 